\title{
INTERPRETATION OF QUASIMOLECULAR L X-RAYS IN HEAVY-ION COLLISIONS ${ }^{\star}$
}

\author{
T. MOROVIĆ, B. FRICKE, W.-D. SEPP \\ Gesamthochschule Kassel, D-3500 Kassel, W. Germany \\ A. ROSÉN \\ Chalmers University of Technology, S-40220 Goeteborg, Sweden \\ and \\ D.E. ELLIS \\ Northwestern University, Evanston, Ill. 60201, USA
}

Received 25 May 1977

\begin{abstract}
Using a relativistic selfconsistent correlation diagram a first interpretation of the shape and position of $\mathrm{L}$ MO X-rays is given within a quasi-adiabatic model.
\end{abstract}

During the past few years the observation of noncharacteristic molecular (MO) X-rays in heavy ionatom collisions became a field of increasing interest $[1-4]$. These X-rays are interpreted as radiative transitions between the time dependent quasi-molecular orbitals built up during the close approach of the two nuclei.

The experimentally observed spectrum so far is an integration over all relative distances and impact parameters. Of particular interest is the $M$ and $L$ noncharacteristic radiation. The observed spectra show a pronounced shoulder (or peak) behaviour thus reflecting a great amount of specific information about the collision process [2-4]. The K MO X-rays on the contrary are all exponentially decreasing continua $[3,4]$.

In a recent paper [5] we gave a detailed interpretation of the structure and threshold behaviour of the M MO X-rays observed in systems like I-Au. Also the threshold behaviour of the $\mathrm{L} X$-rays of $\mathrm{Au}$ has been explained. The interpretation was based on a molecular correlation diagram calculated with a recently developed relativistic Dirac-Slater molecular program [6] . This type of calculation is the most realistic one, because: first the large influence of the electron-electron interaction is fully taken into account, second the relativistic effects (mainly spin-orbit splitting) are large and third, due to selfconsistent effects, the level

\footnotetext{
* Work partially supported by GSI, Darmstadt.
}

behaviour for intermediate distances is very different as compared with all other approximations [7] .

We report in the present work the first interpretation of a L MO X-ray structure exemplified for the $\mathrm{Xe}-\mathrm{Ag}$ system. Such a calculation is a consistency check for the method of interpretation given in ref. [5] because the main contributions of the $M$ and L MO's are explained through different transitions and at different radial distances in the correlation diagrams. The same argument holds true for the connection to specific characteristic X-ray lines. Fig. 1 shows the correlation diagram for the $\mathrm{Xe}-\mathrm{Ag}$ system taking 58 electrons into account. The transitions relevant for the description of the L MO spectrum in this case are those into the $3 \sigma$ to $6 \sigma$ levels as well as the $1 \pi$ level (see fig. 1).

Taking this correlation diagram we have extracted all possible transitions within a broad energetic window where the non-characteristic radiation occurs. The spec trum was calculated by an analytical integration over all impact parameters and a numerical integration over the internuclear distance $R$ as described in the earlier paper [5] . A collision broadening proportional to the slope of the transition energy as a function of $R$ within a Gaussian line shape and a cubic dependence of the transition probabilities on the transition energy were assumed. Of course, the calculation of the occupation numbers as function of $R$ and impact parameter can clearly be performed only by time dependent relativis- 


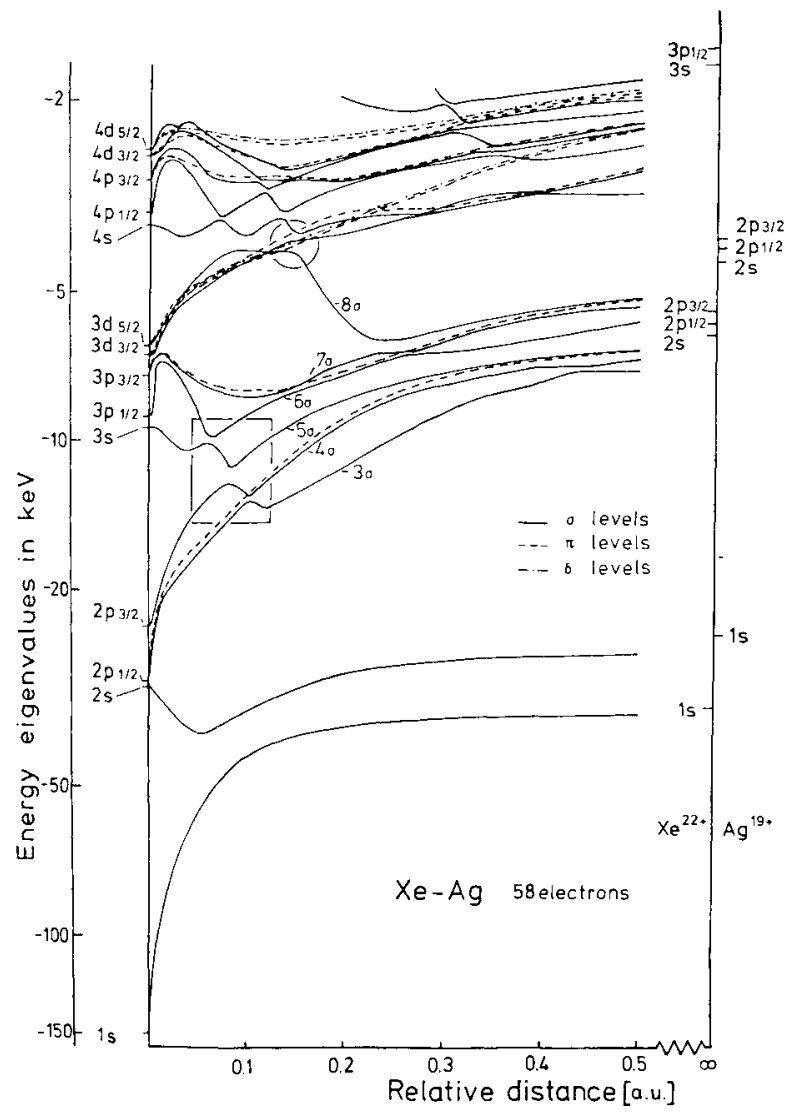

1.ig. 1. Selfconsistent relativistic correlation diagram of the system $\mathrm{Xe}-\mathrm{Ag}$ including 58 electrons. The relevant levels for the MO X-rays are the $3 \sigma-6 \sigma$ and $1 \pi$ levels. Through the encircled crossing the characteristic Ag L X-rays at low impact energies are fed. The crossings within the square distribute the holes into the $\mathrm{L}$ sublevels of $\mathrm{Xc}$.

tic Hartree-Fock calculation which are not available up to now. We therefore assumed here that much more holes will be generated by Coulomb ionization during the first half of the collision process in the $n$ $=3$ levels of the projectile and the target atoms than in the $n=2$ levels. A large percentage of the holes in the $n=3$ levels will be transferred through strong coupling at very small distances into the $n=2$ levels of the system. The pronounced well separated crossings among the $3 \sigma$ to $6 \sigma$ states (shown in the square in fig. 1) allow the use of Landau-Zener type calculations for the occupation numbers as a good approximation. The resulting hole distribution was used in the calculation of the spectrum given in fig. 2 . We get an overall

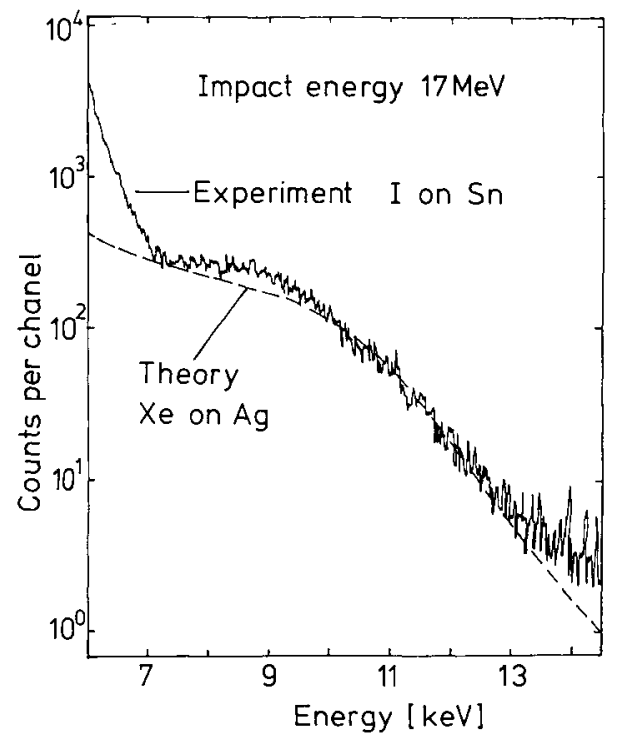

Fig. 2. Experimental X-ray spectrum for $17 \mathrm{MeV}$ I on Sn comparcd with the unnormalized theoretical MO spectrum of Xe on Ag.

good agreement for the L MO X-rays with the available experimental data.

The quality of the calculation of the hole distribution can be checked in the experiment where the relative $\mathrm{L} X$-rays intensities ratio $\left(L_{1}+L_{2}\right) / L_{3}$ from the $M$ shell of the projectile was measured [8]. Our value yields a ratio of about 0.5 whereas the experiment gives 0.4 .

For the impact energies lower than needed to reach the crossings at and below $0.03 \mathrm{au}$, in the vicinity of the united atom $3 \mathrm{~d}$ levels (equivalent to approximately $6 \mathrm{MeV}$ projectile energy), a transfer of holes into $n=2$ levels of the separated atoms should not take place and the L MO X-rays will not be observed anymore. Only the contribution of direct Coulomb ionization of the $n=2$ levels in the projectile and target may still yield a small contribution to the MOX-rays. On the other hand, due to the strange behaviour of the $8 \sigma$ level a transfer of holes in to the $n=2$ states of the separated system is still possible down to less than $2 \mathrm{MeV}$ projectile energy where the two nuclei just reach the encircled crossing at $0.16 \mathrm{au}$. All holes transferred at this crossing will produce characteristic $L$ lines in the lighter atom. The experimental proof of this last prediction will be hard because the projectile energy is already so small that only few electrons will be removed 
by direct Coulomb ionization even in the higher levels. As a conclusion we may say that even by using the integral experimental information of the MO radiation we do learn quite a lot about the complicated system formed during the collision. More details in non-characteristic spectra are expected to be seen in refined coincidence experiments which are under way.

\section{References}

[1] I. Saris, W.F. van der Weg, H. Tawara and R. Laubert, Phys. Rev. Lett. 28 (1972) 717.

[2] P.H. Mokler, H.J. Stein and P. Armbruster, Phys. Rev. Lett. 29 (1972) 827.
[3] Atomic physics, vol. 4, eds. G. zu Putlitz, H.G. Weber and A. Winnacker (Plenum Press, New York 1975), p. 249-324, and references therein.

[4] Proc. of IX Int. Conf. on the Phy sics of electronic and atomic collisions, Seattle, Wash., July 1975; Proc. of Il Int. Conf, on Inner shell ionization phenomena, Freiburg, W. Germany, March 1976.

[5] B. [ricke et al., Phys. Lett. 59A (1976) 375.

[6] A. Rosén and D.E. Ellis, Chem. Phys. Lett. 27 (1974) 595; A. Rosén and D.E. Ellis, J. Chem. Phys. 62 (1975) 3039.

[7] B. Müller, J. Rafelski and W. Greiner, Phys, Lett. 47B (1973) 5 ;

J. Eichler and U. Wille, Phys. Rev. A11 (1975) 1973; K. Taulbjerg and J.S. Briggs, J. Phys. (GB) B8 (1975) 1895.

[8] S. Hagmann, thesis, University of Köln, Köln 1977, and to be published. 